\title{
Multiple Arterial Thrombosis in Seronegative Antiphospholipid Syndrome: Need for New Diagnostic Criteria?
}

\author{
Daniele Pastori ${ }^{1}$, Roberta Misasi ${ }^{2}$, Maurizio Sorice ${ }^{2}$, Francesco Cribari ${ }^{1}$, Danilo Menichelli ${ }^{1}$, Francesco Violi $^{1}$, \\ Pasquale Pignatelli ${ }^{1}$, on behalf of the ATHERO-APS Study Group* \\ ${ }^{1}$ Department of Internal Medicine and Medical Specialties, I Clinica Medica, Atherothrombosis Centre, Sapienza University of Rome, Rome, Italy \\ ${ }^{2}$ Department of Experimental Medicine, Sapienza University of Rome, Rome, Italy
}

Received: 10/06/2019

Accepted: 26/07/2019

Published: 23/09/2019

\begin{abstract}
How to cite this article: Pastori D, Misasi R, Sorice M, Cribari F, Menichelli D, Violi F, Pignatelli P, on behalf of the ATHERO-APS Study Group. Multiple
\end{abstract} arteria thrombosis in seronegative antiphospholipid syndrom: need for a new diagnostic criteria? EJCRIM 2019;6: doi:10.12890/2019_001180.

Conflicts of Interests: The Authors declare that there are no competing interest

Acknowledgements: *Members of the ATHEROsclerosis in Antiphospholipid Syndrome (ATHERO-APS) study group: Marco Antonio Casciaro, Lorenzo Loffredo, Giovanni Talerico, Tommasa Vicario, Gabriele Salvatori.

This article is licensed under a Commons Attribution Non-Commercial 4.0 License

\section{ABSTRACT}

Background: Antiphospholipid syndrome (APS) is defined as thromboembolic complications and/or pregnancy morbidity in the presence of persistent increased titres of antiphospholipid antibodies. Nevertheless, some patients with clinical signs suggestive of APS are negative for diagnostic antibodies and may be classified as having seronegative-APS (SN-APS). Among the 'non-diagnostic' antibodies, a few studies have suggested that the IgG anti-vimentin/cardiolipin antibodies (AVA/CL) may be associated with risk of thrombosis.

Aims: The aim of this case report is to encourage the assessment of non-conventional antibodies in APS.

Patient and methods: We report the case of a 69-year-old male patient with rapid onset of apparently unexplained multiple exclusively arterial thrombotic events in both coronary and peripheral vascular beds.

Results: The patient did not meet the diagnostic criteria for APS but was positive for AVA/CL, which result persisted on further testing at 3 and 6 months.

Discussion: Ongoing research has revealed the existence of non-criteria antibodies which may be relevant for the diagnosis of SN-APS and should be included in the classification criteria for the disease.

\section{LEARNING POINTS}

- In patients with unexplained multiple thrombosis without the conventional antibodies of antiphospholipid syndrome, the assessment of non-conventional antibodies should be considered.

- IgG anti-vimentin/cardiolipin antibodies may be associated with risk of thrombosis.

- Arterial thrombosis could be the only manifestation of antiphospholipid syndrome.

\section{KEYWORDS}

Seronegative antiphospholipid syndrome, vimentin, cardiolipin, antibodies, thrombosis 


\section{of Case Reports in \\ Internal Medicine}

\section{INTRODUCTION}

The clinical presentation of antiphospholipid syndrome may be heterogeneous and multiple organs can be involved. A minority of patients may have clinical signs suggestive of antiphospholipid syndrome but also persistent negative levels of antibodies (cardiolipin antibodies, anti- $\beta 2$ glycoprotein 1 antibodies and lupus anticoagulant). Ongoing research has revealed the existence of non-criteria antibodies which may be relevant for the diagnosis of antiphospholipid syndrome and which could be included in the classification criteria for the disease ${ }^{[1]}$. In this case report, we evaluate anti-vimentin/cardiolipin antibodies (AVA/CL), which, as demonstrated by Ortona et al. ${ }^{[2]}$, mediate activation of the TLR4/IRAK/Nf-kB molecular pathway leading to the release of pro-inflammatory and procoagulant factors by endothelial cells.

AVA/CL antibodies are an intriguing candidate because they are common in seronegative antiphospholipid syndrome (SN-APS) and also seem to exert a pro-thrombotic effect ${ }^{[3]}$. Indeed, antibodies against AVA/CL complex showed a prothrombotic effect and may be involved in arterial thrombosis by inducing platelet and coagulation cascade activation, such as demonstrated in a study conducted on cardiac allografts ${ }^{[4]}$.

\section{CASE REPORT}

A 69-year-old male patient was admitted to the Emergency Department of Umberto I University Hospital Rome for progressive asthenia, abdominal pain with constipation and confusion. His medical history was characterized by ischaemic heart disease treated with triple bypass surgery, recent ischaemic stroke (about 2 months previously), arterial hypertension and benign prostatic hyperplasia. The patient was on chronic medication with aspirin $100 \mathrm{mg} /$ daily, carvedilol $25 \mathrm{mg} /$ daily, atorvastatin $20 \mathrm{mg} /$ daily, dutasteride $0.5 \mathrm{mg} /$ daily and tamsulosin 0.4 $\mathrm{mg} /$ daily.

On physical examination, the patient appeared alert and oriented in time and space and did not show any focal neurological defects.

During his stay in the Emergency Department, the patient experienced epigastric pain and profuse sweating. Electrocardiography showed ST segment elevation in all precordial derivations. High-sensitive troponin T was raised at $0.238 \mu \mathrm{g} / \mathrm{l}$ (normal value $<0.014 \mu \mathrm{g} / \mathrm{I}$ ). Therefore, ASA $250 \mathrm{mg}$, ticagrelor $180 \mathrm{mg}$ and fondaparinux $2.5 \mathrm{mg}$ were administered; percutaneous transluminal coronary angiography was then performed through the left femoral artery, but there were no acute differences from previous angiographic examinations and no endovascular procedures were performed.

Concomitantly with percutaneous transluminal coronary angiography, arterial blood gas analysis from the left brachial artery was performed. After a few hours, the left hand became pale and cold and neither an ulnar nor radial pulse could be detected. Ultrasound and computed tomography angiography (CTA) demonstrated obstruction of the left circumflex humeral artery. The patient was started on unfractionated heparin infusion and aspirin was discontinued. He was then moved from the Emergency Department to the Internal Medicine Ward, where unfractionated heparin infusion was discontinued and enoxaparin 4,000 IU bid was started. Laboratory findings are reported in Table 1.

\begin{tabular}{|l|l|l|}
\hline & Result & Normal Range \\
\hline Creatinine & 0.5 & $0.1-1.2 \mathrm{mg} / \mathrm{dl}$ \\
\hline High-sensitive troponin T & 0.025 & $<0.014 \mathrm{\mu g} / \mathrm{l}$ \\
\hline $\begin{array}{c}\text { Glutamic oxaloacetic transaminase } \\
\text { (GOT) }\end{array}$ & 13 & $8-38 \mathrm{IU} / \mathrm{I}$ \\
\hline $\begin{array}{c}\text { Glutamic-pyruvic transaminase } \\
\text { (GPT) }\end{array}$ & 9 & $12-41 \mathrm{IU} / \mathrm{l}$ \\
\hline Myoglobin & 34 & $28-72 \mathrm{ng} / \mathrm{ml}$ \\
\hline Creatine phosphokinase (CPK) & 50 & $39-308 \mathrm{IU} / \mathrm{I}$ \\
\hline Haemoglobin (HGB) & 14 & $13-17 \mathrm{~g} / \mathrm{dl}$ \\
\hline White blood cell count (WBC) & 9,190 & $4,000-10,000 / \mathrm{mm}^{3}$ \\
\hline Platelets & 231,000 & $150,000-450,000 / \mathrm{mm}^{3}$ \\
\hline International normalized ratio (INR) & 1.14 & $0.8-1.2$ \\
\hline Activated partial thromboplastin \\
time (aPTT ratio) & 0.99 & $0.8-1.2$ \\
\hline Fibrinogen & 282 & $200-400 \mathrm{mg} / \mathrm{dl}^{3}$ \\
\hline C-reactive protein & $<0.1$ & $<0.5 \mathrm{mg} / \mathrm{dl}$ \\
\hline
\end{tabular}

\begin{tabular}{|c|c|c|}
\hline Autoantibodies & Result & Normal Range \\
\hline Anti-nuclear antibodies (ANA) & $\begin{array}{l}\text { Negative } \\
1: 80\end{array}$ & Negative \\
\hline Lupus anticoagulant (LAC, ratio) & 0.92 & $<1.30$ \\
\hline Anti-cardiolipin IgG antibodies & 0.0 & $<15 \mathrm{GPL} / \mathrm{ml}$ \\
\hline Anti-cardiolipin IgM antibodies & 0.0 & $<15 \mathrm{MPL} / \mathrm{ml}$ \\
\hline $\begin{array}{l}\text { Anti- } \beta 2 \text { glycoprotein } 1 \lg G \\
\text { antibodies }\end{array}$ & 0.0 & $<15 \mathrm{UA} / \mathrm{ml}$ \\
\hline $\begin{array}{l}\text { Anti- } \beta 2 \text { glycoprotein } 1 \text { lgM } \\
\text { antibodies }\end{array}$ & 0.1 & $<15 \mathrm{UA} / \mathrm{ml}$ \\
\hline
\end{tabular}

Table 1. Laboratory findings 
During the hospital stay, a pulsatile painless mass was noted in the left femoral area. Arteriography revealed a femoral pseudoaneurysm, which was successfully treated with arteriorrhaphy.

The patient also complained of a painful lower right limb, which appeared pale on physical examination, and no arterial pulse was detectable. CTA demonstrated a thrombotic formation in the right superficial femoral artery extending to the right common femoral artery. This second ischaemic event was effectively treated with thromboendarterectomy.

We considered possible aetiologies for these recurrent arterial thrombotic events. We investigated the panel of autoantibodies listed in Table 1. We found that all conventional autoantibodies required for the diagnosis of antiphospholipid syndrome were negative. We also tested the patient for the most common inherited causes of thrombophilia, such as protein C, protein S, antithrombin III, activated protein $C$ resistance, factor $V$ Leiden, and Factor gene mutation, which were all within normal ranges.

Thus, a diagnosis of SN-APS was made. Further investigation with plasma assay of AVA/CL showed above-normal values (Table 1). At discharge the patient was prescribed vitamin $\mathrm{K}$ antagonists (INR range 2-3) and aspirin $100 \mathrm{mg} /$ daily was restarted. Other medications were unchanged. The patient is undergoing regular follow-up visits and no other thrombotic events have occurred.

This case report describes SN-APS with multiple recurrent arterial thrombosis in a patient without the typical criteria for antiphospholipid syndrome.

\section{DISCUSSION}

Here, we report the case of a patient who developed multiple arterial thrombosis over a very short period in various vascular districts. We made a diagnosis of SN-APS by exclusion, as no other evident cause of arterial thrombosis was found, and all antibodies required for a diagnosis of antiphospholipid syndrome were negative. Interestingly, the positivity for AVA/CL persisted at 3 and 6 months after discharge, along with negativity for all other antibodies.

The role of AVA/CL in SN-APS has been investigated in only one previous study. Ortona et al. ${ }^{[2]}$ analyzed serum IgG AVA/CL antibodies detected by ELISA in 29 SN-APS patients, 40 patients with antiphospholipid syndrome, 30 patients with systemic lupus erythematosus, 30 patients with rheumatoid arthritis, and 32 healthy control patients. They found positivity for AVA/CL in almost all patients with antiphospholipid syndrome (92\%), and also in a large proportion of SN-APS patients (55\%). It was noteworthy that this positivity was persistent in almost all cases.

Anti-vimentin antibodies can be generated upon invasive endovascular procedures, which may represent a local injury to vascular homeostasis triggering an inflammatory response. Thus, in our patient, anti-vimentin antibody formation may have been facilitated by percutaneous transluminal coronary angiography, sampling for arterial blood gas analysis and arteriorrhaphy procedures.

Nevertheless, we cannot exclude the involvement of other autoantibodies not tested in the present study (Table 1 shows a full list of suggested non-conventional antibodies in SN-APS).

All these findings suggest AVA/CL is a potential candidate as an additional diagnostic criterion for SN-APS ${ }^{[5]}$. However, there is not enough evidence to support its routine use in clinical practice, and its assessment should be reserved for patients with no other evident causes of thrombosis.

In conclusion, our case suggests that evaluation of AVA/CL may be helpful in patients with multiple arterial thrombosis who do not fulfil antiphospholipid syndrome criteria.

\section{REFERENCES}

1. Misasi R, Capozzi A, Longo A, Recalchi S, Lococo E, Alessandri C, et al. "New" antigenic targets and methodological approaches for refining laboratory diagnosis of antiphospholipid syndrome. J Immunol Res 2015;2015:858542.

2. Ortona E, Capozzi A, Colasanti T, Conti F, Alessandri C, Longo A, et al. Vimentin/cardiolipin complex as a new antigenic target of the antiphospholipid syndrome. Blood 2010;116(16):2960-2967.

3. Leong HS, Mahesh BM, Day JR, Smith JD, McCormack AD, Ghimire G, et al. Vimentin autoantibodies induce platelet activation and formation of platelet-leukocyte conjugates via platelet-activating factor. J Leukoc Biol 2008;83(2):263-271.

4. Ramzy D, Rao V, Brahm J, Miriuka S, Delgado D, Ross HJ. Cardiac allograft vasculopathy: a review. Can J Surg 2005;48(4):319-327.

5. Lim W, Crowther MA, Eikelboom JW. Management of antiphospholipid antibody syndrome: a systematic review. JAMA 2006;295(9):1050-1057. 